10

\title{
Акустооптическая коммутация волоконно-оптических каналов
}

\author{
() С.Н. Антонов \\ Институт радиотехники и электроники им. В.А. Котельникова РАН, \\ 141190 Фрязино, Московская обл., Россия \\ e-mail: olga-ant@yandex.ru
}

(Поступило в Редакцию 24 мая 2018 г.)

\begin{abstract}
Предложен и создан акустооптический коммутатор волоконно-оптических каналов на базе $\mathrm{TeO}_{2}$ двухкоординатного дефлектора. Коммутатор реализует преключение оптического излучения из одного выходного световода в двумерную входную матрицу световодов, либо обратное - из любого световода матрицы в один выходной световод. Получены значения для основных взаимосвязанных параметров. Установлено, что коммутатор обеспечивает высокое быстродействие $-2-10 \mu \mathrm{s}$, большое число переключаемых каналов - до несколько сотен, небольшие вносимые потери $-2-5 \mathrm{~dB}$, значительную величину развязки между каналами $-35--60 \mathrm{~dB}$. Экспериметы проведены для выходной матрицы с 19 световодами. Показана возможность работы коммутатора в режиме мультипликации каналов - одновременной передачи сигнала из выходного световода в заданное число световодов входной матрицы.
\end{abstract}

DOI: $10.21883 / J T F .2019 .02 .47083 .205-18$

\section{Введение}

Развитие волоконно-оптических систем связи ставит задачу совершенствования оптических средств коммутации: переключателей, мультиплексоров. Современные образцы таких устройств, как правило, основаны на базе электрооптики и микро-электромеханики $[1,2]$. Акустооптические (AO) методы и устройства в этой области изучаются, но пока не находят адекватного использования [3,4]. Существенно, что им присущ ряд весьма немаловажных достоинств: высокая надежность ввиду отсутствия механически перемещаемых элементов, достаточно высокое быстродействие, малые управляющие напряжения и стойкость к интенсивному оптическому излучению.

Работа посвящена разработке АO коммутатора АОК (переключателя) волоконно-оптических каналов (ВОК) и оценке основных практически важных параметров. В работе не рассматриваются конкретные варианты практического использования переключателя. Однако для примера: пусть волоконно-оптическая магистральная линия содержит рабочий волоконный канал и сколько-то резервных. При необходимости смена линии может быть осуществлена за несколько микросекунд.

\section{Принцип функционирования АОК}

Базовый принцип АОК основан на использоваении АО дефлектора (АОД) - устройства, отклоняющего падающий на него оптический луч в различные направления, определяемые частотой управляющего сигнала [5-9].

Алгоритм работы АОК следующий: один выходной канал переключается в $N$ входных $(1 \times N)$ или в силу взаимности АО взаимодействия обратное - один из $N$ выходных каналов переключаются в один входной $(N \times 1)$. Далее для определенности будем рассматривать ситуацию $1 \times N$.
Принцип работы АОК показан на рис. 1.

Свет из выходного волоконного световода (ВС) 1 поступает на коллимирующую систему $L 1$, которая формирует излучение с апертурой $D$ на выходе. Возбуждение акустической волны в АО дефлекторе (АОД) приводит к отклонению света на угол, пропорциональный частоте акустической волны: $\alpha \approx f \lambda / \nu_{s}$, где $\lambda$ - длина волны света, $v_{s}$ - скорость звука, $f$ - частота звука. Отклоненный луч фокусируется линзой $L 2$ на линейку торцов входных ВС 5.

Все расчеты и эксперимены будут проводиться для одномодовых изотропных ВС на длине волны света $1.55 \mu \mathrm{m}$. В качестве АОД использовался анизотропный $\mathrm{AO}$-дефлектор на $\mathrm{TeO}_{2}$ с дифракцией на медленной акустической волне, распространяющейся под углом $6^{\circ}$ к кристаллографической оси $[110]$ со скоростью $0.65 \cdot 10^{6} \mathrm{~mm} / \mathrm{s}[5-9]$.

\section{Двухкоординатный АOK}

Число выходных ВОК многократно увеличивается при использовании двухкоординатной схемы (рис. 2).

При этом за первым АОД стоит второй, ориентированный ортогонально первому. Первая отклоняет свет в одном направлении, а вторая - в ортогональном. Углы отклонения управляются независимо, что позволят адресовать свет в любой ВС выходной двумерной матрицы с общим числом каналов $N_{2 D} \approx N_{1 D}^{2}$, где $N_{1 D}-$ количество каналов, достижимое при использовании только одного дефлектора.

Дифракция света в АОД на кристалле $\mathrm{TeO}_{2}$ происходит для линейно-поляризованного излучения и непосредственная реализация схемы рис. 1 приведет к 50\%-ным потерям для изотропного, не поддерживающего поляризацию ВС (используемого в линиях связи). 
Был создан поляризационно-нечувствительный двухкоординатный дефлектор, принцип работы которого поясняется на рис. 3 [10].

Дефлектор состоит из трех однокоординатных АОД, выполненных на кристаллах $\mathrm{TeO}_{2}$, в каждом из которых дифрагирует свет только определенной поляризации, причем дифрагированный свет имеет поляризацию, ортогональную исходной. Исходное неполяризованное, или произвольно поляризованное излучение 1 в общем случае можно представить в виде двух лучей ортогональных поляризаций, распространяющихся в одном направлении. Поляризации лучей показаны стрелками рядом с номерами. Свет последовательно проходит три АОД. После АОД 1 одна поляризация света проходит без изменений (нулевой порядок дифракции) - луч 2 , а вторая дифрагирует, в результате чего отклоняется и

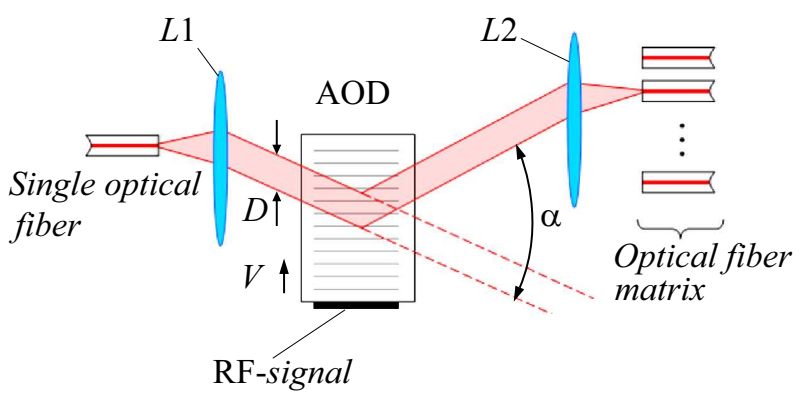

Рис. 1. Схема АО коммутатора.

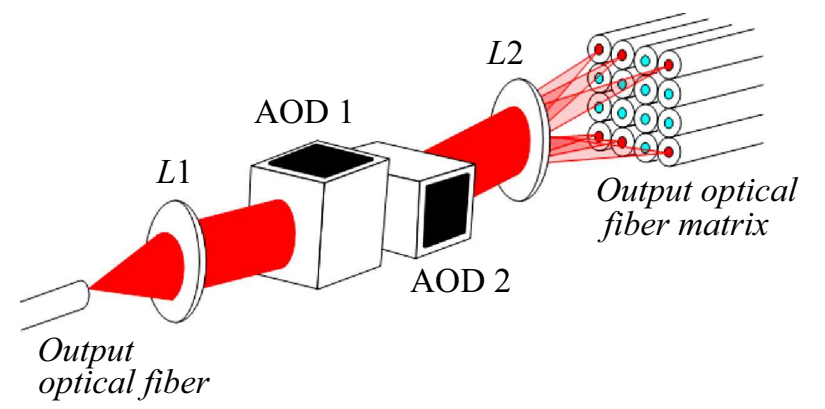

Рис. 2. Принципиальная схема двухкоординатного коммутатора. $L 1$ и $L 2$ - коллимирующая оптика, AOD1 и AOD2 дефлекторы.

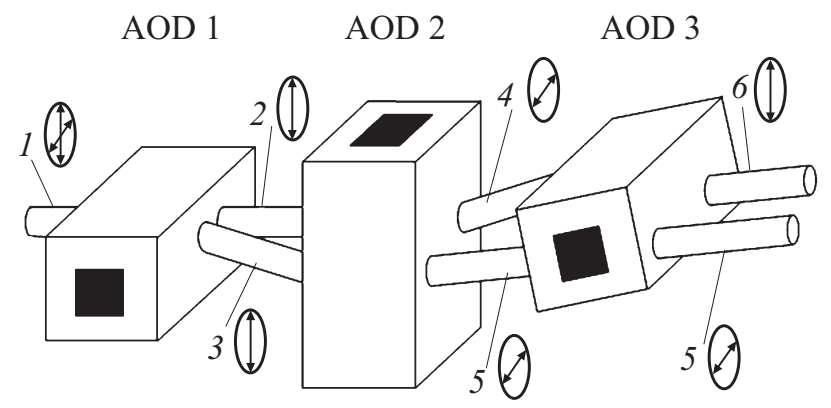

Рис. 3. Схема поляризационно-нечувствительного двухкоординатного дефлектора. AOD1, AOD2 и AOD3 - дефлекторы.

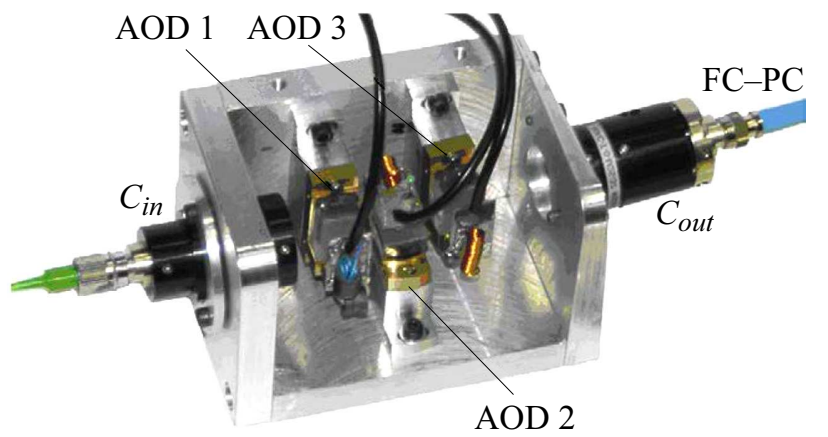

Рис. 4. Внешний вид двухкоординатного коммутатора. $C_{i n}$ и $C_{\text {out }}$ входной и выходной коллиматоры соответственно.

меняет свою поляризацию на противоположную, формируя луч 3. В АОД 2 лучи 2 и 3 испытывают дифракцию и переходят в лучи 4 и 5 соответственно. При этом лучи отклоняются в направлении, перпендикулярном направлению отклонения света в АОД-1. Далее луч 4 дифрагирует в АОД 3, отклоняясь в том же направлении, что и при дифракции в АОД 1 , и образует луч 6 . Луч 5 проходит АОД 3 без дифракции.

Каждая из двух поляризационных составляющих исходного света проходит систему, испытывая два акта дифракции. Дважды поменяв поляризации на ортогональные, эти составляющие на выходе имеют исходные поляризации. Каждая из составляющих при одном акте дифракции отклоняется в одном направлении, а при втором - в ортогональном. На выходе системы образуется излучение с исходной поляризацией, но отклоненное в двух направлениях на независимо управляемые углы, т.е. реализуется двухкоординатное отклонение света произвольной поляризации.

Фотография изготовленного АОК на рис. 4.

АОК включал в себя три АОД, входной и выходной коллиматоры с BC разъемами типа FC-PC. Диаметр коллимированного света в зоне АОД $4.5 \mathrm{~mm}$. Оптические грани $\mathrm{TeO}_{2}$ имели размеры $8 \times 12 \mathrm{~mm}$. Размеры пьезопребразователя из $\mathrm{LiNbO}_{3}: L=5 \mathrm{~mm}$ - длина взаимодействия, $H=6 \mathrm{~mm}$ - высота. Широкий частотный дефлекторов от 12 до $28 \mathrm{MHz}$, обеспечивался аустическим согласованием преобразователя и $\mathrm{TeO}_{2}[11,12]$.

\section{Расчет основных параметров AOK}

Данный раздел посвящен описанию алгоритма расчета основных параметров АОК, определяемых характеристиками АОД.

Рассчитывались:

- потери световой энергии $K$,

- развязка между ВОК - проникновение сигнала из основного канала в - соседние $C$,

- количество каналов $N$,

- быстродействие (время переключения) $\tau$,

- электрическая мощность, подводимая к АОД (определяющая степень нагрева - оптических искажений) $P$. 
Очевидно, что основные параметры АОК находятся в компромиссном соотношении друг с другом. Например, чем больше $N$, тем меньше величина $C$, возрастает $\tau$, увеличивается $P$ и т.п. Таким образом, оптимизация АОК должна производиться при заданных приоритетных параметров. Для определенности, предлагается алгоритм расчета максимального количества каналов, достижимого при заданных значениях остальных параметров.

\section{Быстродействие}

Время переключения АОД $\tau$ задает время, за которое акустическая волна проходит апертуру света $D$, в условиях эксперимента

$$
D[\mathrm{~mm}]=0.65 \cdot \tau[\mu \mathrm{s}] .
$$

При заданном времени переключения $\tau=6 \mu \mathrm{s}$ апертура составляет $D=4 \mathrm{~mm}$.

\section{Пьезопреобразователь и подводимая электри- ческая мощность}

Акустическая мощность, соответствующая максимальной эффективности дифракции, определяется отношением высоты $H$ к длине $L$ пьезопреобразователя (длине взаимодействия). Зависимость приведена на рис. 5.

Подводимая мощность $P_{\text {in }}$ (в силу потерь при преобразовании электрического сигнала в акустический) составляет 1.3-1.7 $P_{a c}$ акустической мощности. Как показывает эксперимент, при мощности более $1.5 \mathrm{~W}$ проявляются тепловые эффекты: кристаллы нагреваются, их оптические свойства изменяются, в результате чего система расстраивается. Так как высота пьезопреобразователя должна быть не меньше чем апертура света $D$, то ограничение $P_{i n}$ задает минимальную величину длины взаимодействия $L$, составляющую (в расчетных условиях) $6 \mathrm{~mm}$.

Все последующие расчеты параметров АОК будут использовать уровень акустической мощности в АО кристалле $1 \mathrm{~W}$, при подводимой $\sim 1.4 \mathrm{~W}$.

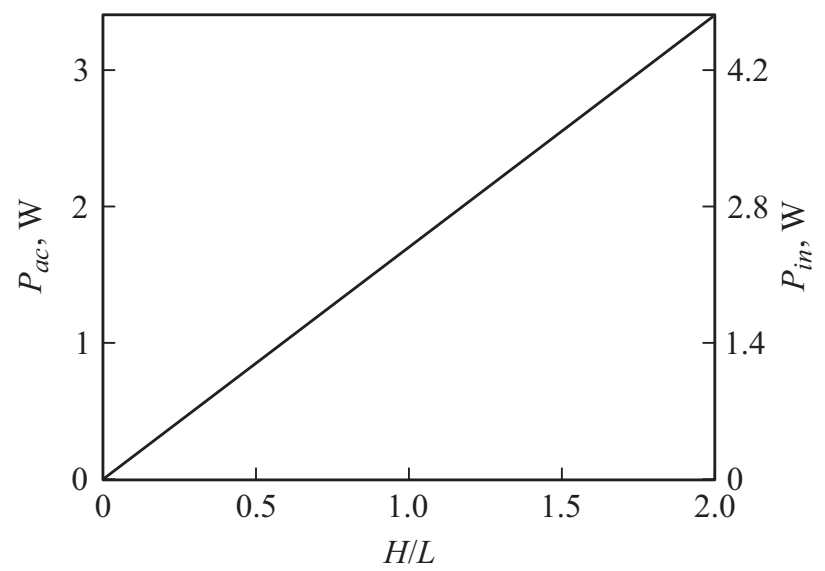

Рис. 5. Подводимая мощность $P_{\text {in }}$ и акустическая мощность $P_{a c}$ в зависимости от соотношения сторон пьезопреобразователя.

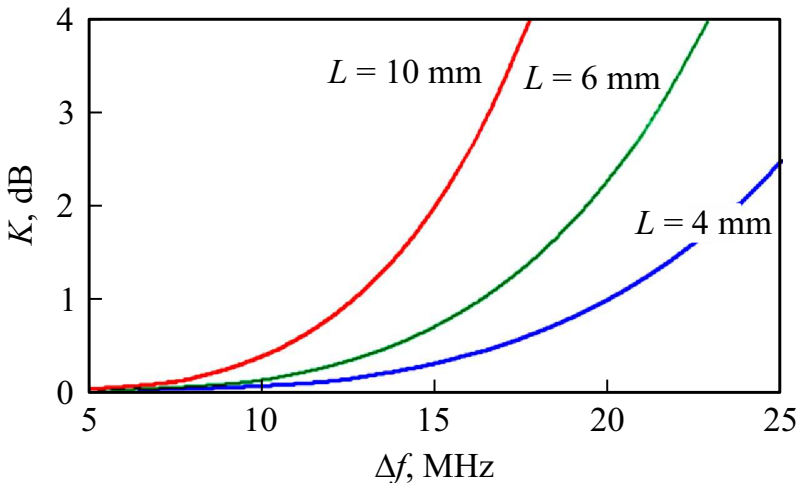

Рис. 6. Связь АО потерь световой энергии с полосой частот АОД.
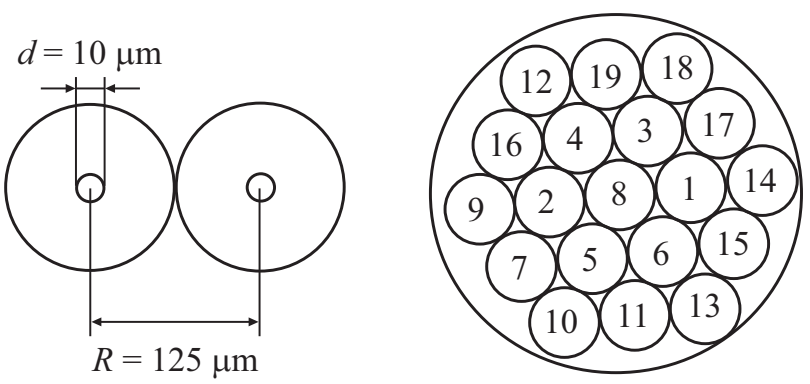

Рис. 7. Схема расположения ВС в матрице.

\section{Акустооптические потери световой энергии}

Важным для оптимизации характеристик АОК является диапазон рабочих частот звука используемых АОД. Расчетная центральная частота звука для рассматриваемого дефлектора равна $20 \mathrm{MHz}$.

Рис. 6 показывает величину потерь $K$, связанных с АО дифракцией (суммарно во всех трех АОД) от полосы частот звука $\Delta f$ для трех длин преобразователя $L(3,6$ и $12 \mathrm{~mm}$ ).

Ограничение величины потерь $K=2 \mathrm{~dB}(63 \%)$ при рассчитанном $L=6 \mathrm{~mm}$ позволяет использовать полосу $\Delta f=18 \mathrm{MHz}$.

Отметим, что здесь оценены только АО потери, связанные со сканированием в определенной полосе частот. Очевидно, будут возникать дополнительные потери, связанные с неоднородностью акустического поля, несовершенством АО кристаллов, неточностью юстировок и т. п. Величина этих дополнительных потерь может колебаться от $0.2 \mathrm{~dB}(95 \%)$ до $0.5 \mathrm{~dB}(90 \%)$. Существенно также, что не учитываются потери при выводе и вводе света из ВС посредством коллиматоров.

\section{Развязка между каналами - переходные зату- хания}

Была изготовлена ВС матрица, состоящая из 19-ти одномодовых ВС (диаметр жилы $10 \mu \mathrm{m}$, оболочки $125 \mu \mathrm{m}$ ), которые монтировались в разъем типа FC-PC с плотной 

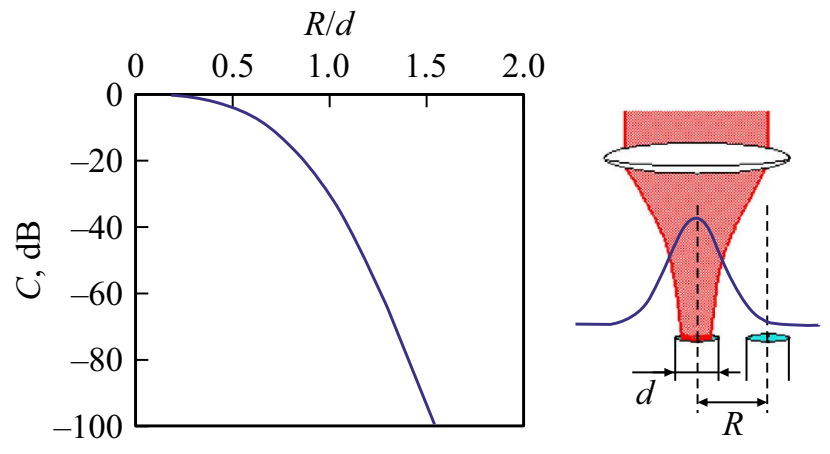

Рис. 8. Связь развязки между соседними ВС и растоянием между ними.

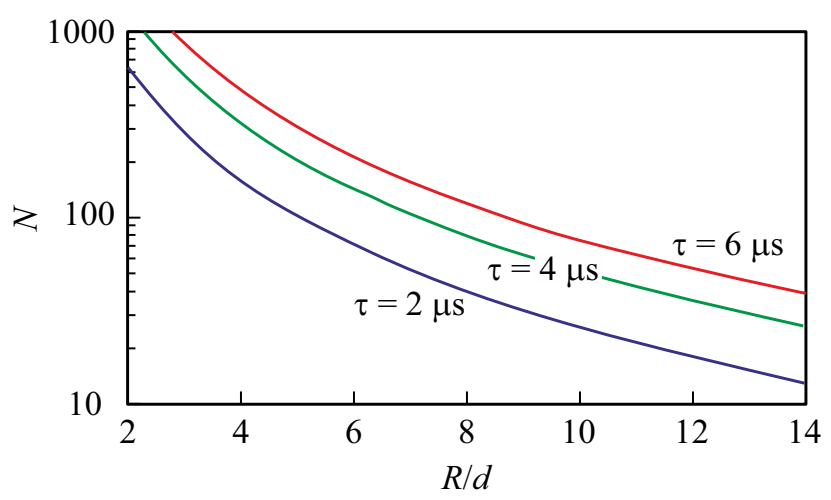

Рис. 9. Число каналов при АО потерях $K=1 \mathrm{~dB}$.

гексагональной упаковкой $(R / d \approx 12)$. После вклейки ВС в разъем производилась оптическая полировка торцов. Схема расположения ВС в матрице и их нумерация показана на рис. 7.

Паразитное проникновение света в нерабочие каналы определяется пространственным распределением световой мощности в плоскости матрицы ВС. Величина этого проникновения полностью определяется плотностью упаковки ВС в матрице, т.е. отношением расстояния между соседними волокнами $R$ к диаметру световедущей жилы волокна $d$. Чем дальше отстоят друг от друга ВС, тем меньше засветка, т.е. заданная величина развязки определяет предельную плотность упаковки ВС в матрице.

Развязка, которую дает идеализированный гауссовый световой луч, показана на рис. 8.

Очевидно, что практическая ситуация значительно хуже. Это связано с большими „фоновыми“ засветками излучения на выходе ВС (например, это оболочечные моды), а также с рассеянием на оптических поверхностях. Как будет показано ниже, при плотности упаковки $\mathrm{BC}$ с $R / d=12$ (эксперимент) величина $C$ не хуже $45 \mathrm{~dB}$.

\section{Число каналов}

Выбранные быстродействие, полоса рабочих частот и плотность упаковки ВС в матрице определяют макси- мальное количество каналов:

$$
N=((d / R) \cdot \Delta f[M h z] \cdot \tau[\mu \mathrm{s}])^{2} .
$$

Для примера, рассмотрим экспериментальную ситуацию: стандартные ВС с диаметром оболочки $125 \mu \mathrm{m}$ и жилы $10 \mu \mathrm{m}$. Графическое отражение данной зависимости приведено на рис. 9, где показано, как связано предельное число ВС каналов от плотности упаковки матрицы при трех величинах быстродействия - 2, 4 и $6 \mu$ s. Расчет проведен при потерях $K=1 \mathrm{~dB}$ (пропускание $80 \%$ ).

Анализируя рис. 9, можно выбрать комбинацию параметров АОК, удовлетворяющую конкретным требованиям.

\section{Экспериментальный макет AOK, результаты измерений}

Рис. 10 представляет внешний вид созданного АОК совместно с входным ВС и выходными 19 ВС.

\section{Потери световой энергии}

За величину потерь, равную $K=0 \mathrm{~dB}$ (100\%), был принят сигнал, который поступает на фотоприемник при непосредственном соединении ВС от лазерного модуля к ВС фотоприемника, минуя коллиматоры.

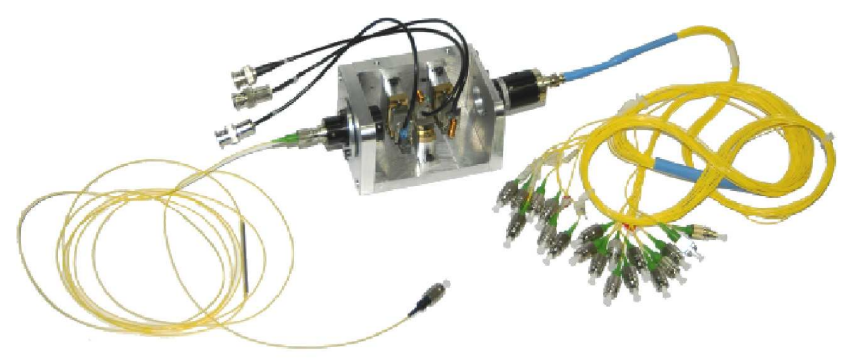

Рис. 10. АОК в сборе с входным и выходными ВС.

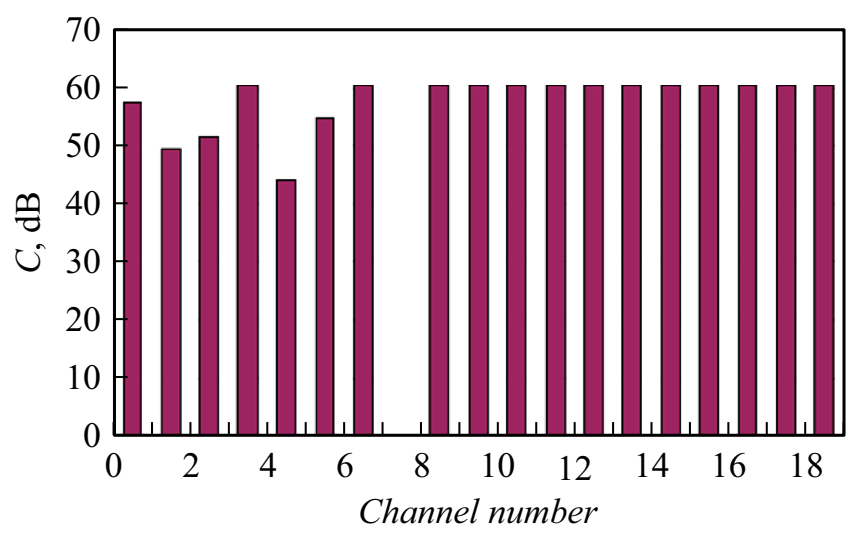

Рис. 11. Величина развязки между каналами. 
Результаты измерений:

a) потери коллиматор - коллиматор без АО кристаллов: $-1.8 \mathrm{~dB}(67 \%)$,

б) потери коллиматор - коллиматор с АО кристаллами (без звука): $-3 \mathrm{~dB}(50 \%)$,

в) суммарные потери в режиме работы АОК: от $3.8 \mathrm{~dB}$ (42\%) до $5.3 \mathrm{~dB}(30 \%)$.

Таким образом, как видно, значительный вклад в величину потерь вносит ввод и вывод света из ВС и обратно посредством данных коллиматоров. В то же время дифракция на АОЯ вносит потери около $2 \mathrm{~dB}$ $(63 \%)$.

\section{Развязка между каналами}

Под величиной развязки между каналами будем понимать отношение световой энергии на выходе данного (рабочего) ВС к энергии на выходе любого другого ВС. Динамический диапазон установки составил $60 \mathrm{~dB}$.

Рисунок 11 - величина развязки между каналами C, когда рабочим каналом являлся 8-й (центральный) ВС матрицы.

Видно, что для близлежащих ВС (номера 2,3,5,6) имеют развязку в пределах от -45 до $-55 \mathrm{~dB}$, а для периферийных ВC $-60 \mathrm{~dB}$.

Следовательно, данная степень упаковки ВС в матрице с соотношением $R / d=12.5$ обеспечивает развязку каналов не более $-35 \mathrm{~dB}$.

\section{Мультипликация BOK}

Мультипликация ВОК - одновременная передача сигнала из выходного ВС в заданное число ВС входной матрицы. Возможность такой работы АОД основана на многолучевой дифракции $[13,14]$. При этом дифракционное поле имеет вид нескольких самостоятельных близкорасположенных по углу лучей равной интенсивности с суммарной эффективностью, близкой к 100\%. Такая $\mathrm{AO}$ дифракция возникает, если управляющий сигнал является периодическим с периодом модуляции $T$ в виде суммы частотных компонент, расположенных с шагом частоты $2 \pi / T$ :

$$
U(t)=\operatorname{Re}\left[\sum_{n} a_{n} \exp \left(-2 \pi j f_{0} t-j \frac{2 \pi n}{T} t+j \varphi_{n}\right)\right]
$$

где $a_{n}-$ амплитуды частотных компонент, $j-$ мнимая единица, $f_{0}$ - несущая частота, $\varphi-$ фазы частотных компонент.

Рис. 12 иллюстриует работу АОК как мультиплексора.

Двумерное распределение интенсивности света в плоскости матрицы выходных ВС является произведением двух одномерных распределений по осям $X$ и $Y$, создаваемых каждой из АОД по отдельности. Существенно, что исходное (входное) излучение делится на лучи равной мощности.

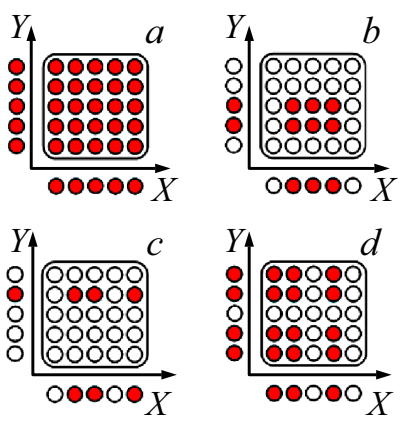

Рис. 12. Схема работы двухкоординатного АОД как мультиплексора.

Некоторые примеры распределений представлены на рис. 12. Здесь схематично изображена матрица ВС. Элементы матрицы, в которые направляются лучи света, закрашены. По осям в таком же виде изображены некоторые комбинации лучей, создаваемые каждой из АОЯ по отдельности. Показаны следующие варианты мультиплицирования:

a) во все выходные ВС одновременно,

b) в произвольную прямоугольную область матрицы,

c) в любой набор ВС одной строки или одного столбца матрицы,

d) в ВC, находящиеся на пересечении набора строк с набором столбцов матрицы.

\section{Выводы}

Разработана концепция, алгоритм расчета параметров и создан экспериментальный макет акустооптического коммутатора волоконно-оптических каналов на базе $\mathrm{TeO}_{2}$ двухкоординатного дефлектора. Коммутатор реализует алгоритм: один выходной канал переключается в $N$ входных $(1 \times N)$ или в силу взаимности АО взаимодействия обратное: $N$ выходных каналов переключаются в 1 входной $(N \times 1)$. Получено графическое отображение основных взаимосвязанных параметров. Акустооптический коммутатор имеет ряд преимуществ по сравнению с электрооптическими и основанными на технологии микро-электромеханики. Расчеты и экспериментальные измерения показали: коммутатор обеспечивает: высокое быстродействие - 2-10 $\mathrm{s}$, принципиально большое число каналов - до несколько сотен, небольшие суммарные потери - $2-5 \mathrm{~dB}$, значительная величина развязки между каналами - 35--60 dB. Показана возможность работы коммутатора в режиме мультипликации каналов - одновременной передачи сигнала из выходного световода в заданное число световодов входной матрицы.

Автор выражает благодарность к.ф.-м.н. Вайнеру А.В. за помощь в расчетах и графическом отображении результатов. 


\section{Список литературы}

[1] Guerre R., Fahrni F., Renaud P. // J. Ligtwave Technol. 2006. Vol. 24. N 3. P. 1486-1498.

[2] Muller H., Chiow S.W., Herrmann S., Chu S. // Rev. Scient. Instrum. 2007. Vol. 78. N 12. P. 124702.

[3] Harris D.O., Vanderlugt A. // Appl. Opt. 1992. Vol. 31. N 11. P. $1684-1686$.

[4] Paparao P., Boothroyd S.A., Robertson W.M. et al. // Appl. Opt. 1994. Vol. 33. N 11. P. 2140-2146.

[5] Yano T., Kawabuichi M., Fukumoto A., Watanabe A. // Appl. Phys. Lett. 1975. Vol. 26. N 12. P. 689.

[6] Магдич Л.Н., Молчанов В.Я. Акустооптические устройства и их применение. М.: Советское радио, 1978. 111 с.

[7] Антонов С.Н., Вайнер А.В., Проклов В.В., Резвов Ю.Г. // ЖТФ. 2013. Т. 83. Вып. 9. С. 108-113.

[8] Антонов С.Н. // ЖТФ. 2016. Т. 86. Вып. 1. С. 136-139.

[9] Антонов С.Н. // ЖТФ. 2016. Т. 86. Вып. 10. С. 155-158.

[10] Антонов С.Н., Вайнер А.В. Способ двухкоординатного отклонения оптического излучения. Патент RU $2355007 \mathrm{C} 1$.

[11] Антонов С.Н., Таешников А.Б. // Акуст. журн. 1991. Т. 37. № 5. C. $837-842$.

[12] Антонов С.Н. // Акуст. журнал. 2017. Т. 63. № 4. C. $364-370$.

[13] Антонов С.Н. // ЖТФ. 2005. Т. 75. Вып. 4. С. 122-124.

[14] Антонов С.Н., Резвов Ю.Г. // ЖТФ. 2007. Т. 77. Вып. 8. C. $93-100$. 